\title{
The clinical and genetic features of COPD-asthma overlap syndrome
}

\author{
Megan Hardin ${ }^{1,2}$, Michael Cho ${ }^{1,2}$, Merry-Lynn McDonald ${ }^{1}$, Terri Beaty ${ }^{3}$, \\ Joe Ramsdell ${ }^{4}$, Surya Bhatt ${ }^{5}$, Edwin J.R. van Beek ${ }^{6}$, Barry J. Make?, \\ James D. Crapo ${ }^{7}$, Edwin K. Silverman ${ }^{1,2}$ and Craig P. Hersh ${ }^{1,2}$
}

Affiliations: 'Channing Division of Network Medicine, Brigham and Women's Hospital, Harvard Medical School, Boston, MA, USA. 'Division of Pulmonary and Critical Care Medicine, Brigham and Women's Hospital, Harvard Medical School, Boston, MA, USA. ${ }^{3}$ Dept of Epidemiology, Johns Hopkins University, Baltimore, MD, USA. ${ }^{4}$ Dept of Medicine, University of California San Diego, La Jolla, CA, USA. ${ }^{5}$ Dept of Pulmonary, Allergy and Critical Care Medicine, University of Alabama, Birmingham, AL, USA. ${ }^{6}$ Clinical Research Imaging Centre, University of Edinburgh, Edinburgh, UK. ${ }^{7}$ Dept of Medicine, National Jewish Health, Denver, CO, USA

Correspondence: Megan Hardin, Channing Division of Network Medicine, Brigham and Women's Hospital, 181 Longwood Ave, Boston, MA 02116, USA. E-mail: remehQchanning.harvard.edu

ABSTRACT Individuals with chronic obstructive pulmonary disease (COPD) and asthma are an important but poorly characterised group. The genetic determinants of COPD and asthma overlap have not been studied. The aim of this study was to identify clinical features and genetic risk factors for COPD and asthma overlap.

Subjects were current or former smoking non-Hispanic whites or African-Americans with COPD. Overlap subjects reported a history of physician-diagnosed asthma before the age of 40 years. We compared clinical and radiographic features between COPD and overlap subjects. We performed genome-wide association studies (GWAS) in the non-Hispanic whites and African-American populations, and combined these results in a meta-analysis. More females and African-Americans reported a history of asthma. Overlap subjects had more severe and more frequent respiratory exacerbations, less emphysema and greater airway wall thickness compared to subjects with COPD alone.

The non-Hispanic white GWAS identified single nucleotide polymorphisms in the genes CSMD1 ( rs 11779254, $\left.\mathrm{p}=1.57 \times 10^{-6}\right)$ and SOX5 $\left(\mathrm{rs} 59569785, \mathrm{p}=1.61 \times 10^{-6}\right)$ and the meta-analysis identified single

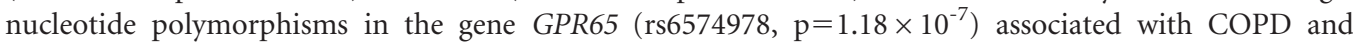
asthma overlap.

Overlap subjects have more exacerbations, less emphysema and more airway disease for any degree of lung function impairment compared to COPD alone. We identified novel genetic variants associated with this syndrome. COPD and asthma overlap is an important syndrome and may require distinct clinical management.

@ERSpublications

We identified distinct clinical features and possible genetic risk factors for subjects with both COPD and asthma http://ow.ly/uWWBc

For editorial comments see page 277.

This article has supplementary material available from erj.ersjournals.com

Received: Dec 112013 | Accepted after revision: March 022014 | First published online: May 292014

Support statement: This study was funded by the following grants from the National Institutes of Health (Bethesda, MD, USA): T32 HL007427-31 (M. Hardin), R01HL089856 (E.K. Silverman), R01HL089897 (J.D. Crapo), R01NR013377 (C.P. Hersh), R01HL094635 (C.P. Hersh) and P01HL083069 (E.K. Silverman). The COPDGene project is supported by grant awards from the National Heart, Lung, and Blood Institute. The COPDGene project is also supported by the COPD Foundation through contributions made to an industry advisory board comprised of AstraZeneca, Boehringer Ingelheim, Novartis, Pfizer, Siemens and Sunovion. The content of this manuscript is solely the responsibility of the authors. None of the above named entities participated in the design or conduct of the study, the collection, management, analysis or interpretation of the data, or the preparation, review, approval or decision to submit the manuscript for publication.

Conflict of interest: Disclosures can be found alongside the online version of this article at erj.ersjournals.com 


\section{Introduction}

The presence of asthma among subjects with chronic obstructive pulmonary disease (COPD) is well recognised, and up to $24 \%$ of patients with COPD will additionally report a history of asthma [1]. However, few studies have investigated the clinical features of this overlap syndrome, and none have investigated the genetics of this syndrome. The dual diagnosis of COPD and asthma is often an exclusion criterion in most studies investigating these diseases individually, limiting the large-scale cohorts available for investigation. Despite this, there is growing evidence that subjects with both COPD and asthma represent an important and distinct patient population, with worse clinical features than those with COPD alone [2, 3]. In recognition of this important subset of patients, the Spanish, Canadian and Japanese respiratory societies have recently included the identification and management of these subjects in their COPD guidelines [4-6].

Both COPD and asthma are heritable diseases with known genetic risk factors. Recent candidate gene and genome-wide association studies (GWAS) have identified genetic variants associated with COPD and asthma individually, and several of these variants have been associated with both diseases in candidate gene studies $[7,8]$. However, no genetic studies have been performed in this overlap group that has both COPD and asthma.

In a previous study, our group investigated the clinical features of the overlap population in the first 2500 subjects enrolled in the COPDGene study [3]. In that analysis, we demonstrated that overlap subjects had more respiratory exacerbations despite fewer pack-years of smoking. The COPDGene study has now completed enrolment, with $>10000$ subjects enrolled. We took advantage of this larger data set, which includes quantitative chest computed tomography (CT) analysis and genome-wide genotyping, to better characterise the clinical, radiographic and genetic features of this overlap group. We hypothesised that this larger cohort of subjects with more complete CT phenotyping and genetic data would allow us to expand on the clinical features we had previously identified. Specifically, radiographic features would demonstrate that this is a pathologically distinct clinical group, and our genetic analysis would identify unique genetic risk factors.

\section{Methods}

Subjects

The subjects from COPDGene were non-Hispanic white or African-American current or former smokers aged 45-80 years with at least a 10 pack-year history of smoking [9]. All subjects completed study questionnaires, performed standardised spirometry and had volumetric chest CT scan at full inspiration and relaxed expiration. Study protocols are available on the COPDGene website (www.copdgene.org). Bronchodilator responsiveness was defined as having a post-bronchodilator improvement in forced expiratory volume in $1 \mathrm{~s}$ (FEV1) of $>200 \mathrm{~mL}$ and 12\% [10]. Quantitative bronchodilator response was measured as the absolute difference between post-bronchodilator FEV1 and pre-bronchodilator FEV1. Maternal and paternal asthma were self-reported. Hay fever history was based on self-reporting of physician-diagnosed hay fever. Exacerbations were defined as the number of respiratory exacerbations experienced in the year prior to enrolling in COPDGene, and severe exacerbations were defined as a history of respiratory exacerbations that resulted in an emergency room visit or hospital stay. Quantitative emphysema was defined as the percentage of lung area with low attenuation areas $<-950$ Hounsfield units (HU) using SLICER software (www.slicer.org) [11]. Airway wall thickening was measured as the square root of wall area of a $10-\mathrm{mm}$ luminal perimeter (Pi10) [12]. Airway wall thickening and segmental and subsegmental wall area were quantified using VIDA software (VIDA Diagnostics, Coralville, IA, USA) as previously described [13]. Percentage of gas trapping was defined as the percentage of lung voxels with attenuation $<-856 \mathrm{HU}$ on expiratory CT imaging $[9,14]$. Subjects provided written informed consent, and the COPDGene study was approved by the institutional review boards at Partners Healthcare (Boston, MA, USA) and all participating centres.

\section{Statistical analysis}

This analysis was restricted to COPD subjects with Global Initiative for Chronic Obstructive Lung Disease (GOLD) stage 2 or greater ( $\mathrm{FEV} 1 /$ forced vital capacity $<0.7, \mathrm{FEV} 1<80 \%$ predicted). Overlap subjects are defined as COPD patients who additionally reported a history of asthma that was diagnosed by a physician before the age of 40 years. These overlap subjects were compared to subjects with COPD alone. We performed an additional sensitivity analysis comparing subjects with COPD to those with COPD and atopic asthma, defined by a physician's diagnosis of hay fever in addition to asthma. Logistic and linear regression models were used to compare binary and quantitative measures of disease, respectively, adjusting for age, race, sex and pack-years of smoking. CT measures of emphysema were additionally adjusted for current smoking status and, along with wall thickness, were additionally adjusted for CT scanner model and body mass index (BMI). We performed a parallel analysis excluding the original 915 subjects who participated in 
the initial study (online supplementary material), as well as an analysis including all subjects with COPD GOLD stage 1-4.

\section{Genetic analysis}

All subjects were genotyped on the Illumina HumanOmniExpress array (Illumina, San Diego, CA, USA). All single nucleotide polymorphisms (SNPs) passed quality control as described previously [15]. Additional imputation of over six million SNPs was performed using $\mathrm{MaCH}$ and minimac $[16,17]$, with the 1000 Genomes Phase I v3 European reference panel for the non-Hispanic white subjects and cosmopolitan reference panels for African-American subjects. This resulted in over six million SNPs for analysis. Due to our small sample size and lack of an available replication population, we removed all SNPs with minor allele frequency $<5 \%$ and imputed SNPs with $\mathrm{r}^{2}<0.80$, leaving 5333124 non-Hispanic white SNPs and 6593020 African-American SNPs for analysis. Principal components for genetic ancestry were generated for nonHispanic white and African-American subjects separately, using the programme EIGENSOFT 2.0 [18].

PLINK was used for genome-wide association analysis of autosomal SNPs in a case-control analysis [19], comparing subjects with COPD and asthma to subjects with COPD alone, adjusted for age, sex and packyears, as well as population-defined principal components for genetic ancestry. GWAS testing incorporated both genotyped and imputed SNPs under an additive genetic model. GWAS was performed separately in the non-Hispanic white and African-American subjects, and a fixed-effects meta-analysis was completed to combine these two groups using METAL [20]. We used PLINK to test for linkage disequilibrium and SNAP to present regional association plots of our most significant associations [21]. We additionally investigated previously identified asthma SNPs from the Gabriel and EVE consortia [22, 23], as well as 10 SNPs previously associated with COPD (online supplementary material) [24].

\section{Results}

\section{Clinical characteristics}

Subject demographics are presented in table 1. Subjects with COPD and asthma were younger (60 years versus 64 years, $\mathrm{p}<0.001)$, had a higher BMI $\left(28.2 \mathrm{~kg} \cdot \mathrm{m}^{-2}\right.$ versus $\left.27.9 \mathrm{~kg} \cdot \mathrm{m}^{-2}, \mathrm{p}=0.006\right)$ and had fewer pack-years of smoking (mean \pm SD $45.7 \pm 25.1$ pack-years versus $54.2 \pm 27.8$ pack-years, $\mathrm{p}<0.001$ ). There were a greater percentage of females $(56 \%$ versus $43 \%, \mathrm{p}<0.001)$ and African-Americans ( $37 \%$ versus $20 \%$, $\mathrm{p}=0.006)$ in the overlap group compared to the COPD group. There was no difference in the percentage of subjects with a positive bronchodilator response or in absolute bronchodilator response between the two groups.

In multivariate analyses adjusting for age, race, sex and pack-years of smoking, we compared clinical features and risk factors between the overlap subjects and those with COPD alone (table 2). Overlap subjects had worse measures of disease severity than those with COPD alone, including higher BODE (BMI, airflow obstruction, dyspnoea and exercise capacity score) (mean \pm SD $3.1 \pm 2.0$ versus $2.9 \pm 2.1, \mathrm{p}=0.02$ ) and higher St George's Respiratory Questionnaire scores $(47.4 \pm 22.7$ versus $39.7 \pm 21.5, \mathrm{p}<0.001)$. Overlap subjects had more exacerbations per year $(1.2 \pm 1.6$ versus $0.7 \pm 1.2, \mathrm{p}<0.001)$ and a greater percentage of

\begin{tabular}{lccc}
\hline TABLE 1 Subject characteristics & & & \\
& COPD & COPD and asthma & p-value \\
\hline Subjects $\mathbf{n}$ & 3120 & 450 & \\
Age years & $64.0 \pm 8.4$ & $60.0 \pm 8.7$ & $<0.001$ \\
Females & $1335(42.8)$ & $252(56)$ & $<0.001$ \\
African-American & $627(20.1)$ & $167(37.1)$ & $<0.001$ \\
Pack-years & $54.2 \pm 27.8$ & $45.7 \pm 25.1$ & $<0.001$ \\
BMI kg m $^{-2}$ & $27.9 \pm 6.1$ & $28.8 \pm 6.9$ & 0.006 \\
FEV1 L & $1.45 \pm 0.63$ & $1.40 \pm 0.62$ & 0.16 \\
FEV1 \% predicted & $50.3 \pm 18.0$ & $50.3 \pm 17.9$ & 0.95 \\
FEV1/FVC & $0.49 \pm 0.13$ & $0.51 \pm 0.13$ & 0.02 \\
Emphysema & $13.54 \pm 12.95$ & $9.93 \pm 11.5$ & $<0.001$ \\
Bronchodilator responsiveness & $1120(36.13)$ & $177(39.42)$ & 0.19 \\
Absolute BDR L & $0.09 \pm 0.16$ & $0.11 \pm 0.16$ & 0.11 \\
\hline
\end{tabular}

Data are presented as mean \pm SD or $\mathrm{n}(\%)$, unless otherwise stated. COPD: chronic obstructive pulmonary disease; BMI: body mass index; FEV1: forced expiratory volume in $1 \mathrm{~s}$; FVC: forced vital capacity; BDR: bronchodilator response (>200 $\mathrm{mL}$ and $12 \%$ change in FEV1). 
TABLE 2 Clinical features of subjects with chronic obstructive pulmonary disease (COPD) and asthma compared to those with COPD alone

\begin{tabular}{lcccc} 
& COPD & $\begin{array}{c}\text { COPD and } \\
\text { asthma }\end{array}$ & Effect size & p-value \\
\hline Females & $1335(42.8)$ & $252(56.0)$ & $1.59(1.29-1.95)$ & $<0.001$ \\
African-American & $627(20.1)$ & $167(37.1)$ & $1.74(1.39-2.18)$ & $<0.001$ \\
Bronchodilator responsiveness & $1120(36.1)$ & $177(39.4)$ & $1.19(0.97-1.47)$ & 0.10 \\
Absolute BDR L & $0.09 \pm 0.16$ & $0.11 \pm 0.16$ & $0.02 \pm 0.008$ & 0.03 \\
BODE score & $2.9 \pm 2.1$ & $3.1 \pm 2.0$ & $0.25 \pm 0.1$ & 0.02 \\
SGRQ score & $39.7 \pm 21.5$ & $47.4 \pm 22.7$ & $6.81 \pm 1.1$ & $<0.001$ \\
Exacerbations per year & $0.7 \pm 1.2$ & $1.2 \pm 1.6$ & $0.56 \pm 0.06$ & $<0.001$ \\
Severe exacerbations & $646(20.7)$ & $153(34.0)$ & $1.70(1.36-2.12)$ & $<0.001$ \\
Hay fever & $442(17.8)$ & $186(50.3)$ & $4.66(3.68-5.90)$ & $<0.001$ \\
High school graduates & $1828(58.6)$ & $261(58.0)$ & $1.10(-0.19-0.39)$ & 0.54 \\
Maternal asthma & $162(7.0)$ & $57(19.0)$ & $2.22(1.59-3.12)$ & $<0.001$ \\
Paternal asthma & $123(5.9)$ & $47(17.5)$ & $2.64(1.82-3.83)$ & $<0.001$ \\
\hline
\end{tabular}

Data are presented as $\mathrm{n}(\%)$ or mean \pm SD, unless otherwise stated. Effect size data are presented as OR $195 \%$ CI) or $\beta \pm$ SE. Linear and logistic regression models were adjusted for age, race, sex and pack-years. BDR: bronchodilator response (>200 $\mathrm{mL}$ and $12 \%$ change in forced expiratory volume in $1 \mathrm{~s}$ ); BODE: body mass index, airflow obstruction, dyspnoea and exercise capacity; SGRQ: St George's Respiratory Questionnaire.

these subjects had severe exacerbations resulting in an emergency room visit or hospital stay in the previous year $(34.0 \%$ versus $20.7 \%, \mathrm{p}<0.007)$. These subjects were more likely than those with COPD alone to have a history of hay fever that was diagnosed by a physician $(50.3 \%$ versus $17.8 \%, \mathrm{p}<0.001)$, and were more likely to have a mother $(19 \%$ versus $7 \%, \mathrm{p}<0.001)$ or father $(17.5 \%$ versus $5.9 \%, \mathrm{p}<0.001)$ with asthma. Overlap subjects had a significantly greater quantitative increase in FEV1 in response to bronchodilators $\left(0.11 \pm 0.16 \mathrm{~L} \cdot \mathrm{s}^{-1}\right.$ versus $\left.0.09 \pm 0.16 \mathrm{~L} \cdot \mathrm{s}^{-1} ; \beta \pm \mathrm{SE} 0.02 \pm 0.008, \mathrm{p}=0.03\right)$; however, these differences were clinically minimal. These results were similar when comparing subjects with COPD to those with COPD and atopic asthma (table S1). We performed a parallel analysis including only those subjects who were not included in the first analysis [3], and these findings remained significant (table S2).

In addition, we performed a sensitivity analysis in subjects with GOLD stage 1-4 COPD comparing the overlap subjects to those with COPD alone. In this analysis, overlap subjects demonstrated no difference in radiographic emphysema (COPD and asthma $1.36 \pm 1.54 \%$ versus COPD $1.74 \pm 1.45 \%$; $\beta \pm$ SE $-0.07 \pm 0.07$, $\mathrm{p}=0.33$ ). The remainder of the analysis was similar to the primary analysis in GOLD $2-4$ subjects (data not shown).

As the COPD and asthma subjects demonstrated higher BMI, we performed an analysis additionally adjusting for BMI. Adjusting for BMI strengthened the association between the overlap syndrome and higher BODE score $(\beta \pm$ SE $0.28 \pm 0.11, p=0.008)$ and had minimal impact on the number of exacerbations per year $(\beta \pm$ SE $0.55 \pm 0.06, \mathrm{p}<0.001$ ) or the odds ratio for severe exacerbations (OR 1.70 (95\% CI 1.36-2.13), $\mathrm{p}<0.001$ ) and did not significantly impact on the associations from the remainder of the analysis (data not shown).

TABLE 3 Chest computed tomography scan features of subjects with chronic obstructive pulmonary disease (COPD) and asthma compared to those with COPD alone

\begin{tabular}{lcccc} 
& COPD & COPD and asthma & Effect size & p-value \\
\hline Log emphysema\# \% & $1.91 \pm 1.4$ & $1.44 \pm 1.6$ & $-0.23 \pm 0.07$ & $<0.001$ \\
Pi10 mm & $3.71 \pm 0.14$ & $3.78 \pm 0.16$ & $0.06 \pm 0.01$ & $<0.001$ \\
Segmental airway wall area \% & $62.8 \pm 3.0$ & $63.6 \pm 3.3$ & $0.61 \pm 0.16$ & $<0.001$ \\
Subsegmental airway wall area \% & $65.6 \pm 2.3$ & $66.4 \pm 2.7$ & $0.66 \pm 0.20$ & 0.001 \\
Gas trapping \% & $39.7 \pm 20.7$ & $35.6 \pm 21.5$ & $0.88 \pm 1.5$ & 0.55 \\
\hline
\end{tabular}

Data are presented as mean \pm SD, unless otherwise stated. Effect size data are presented as $\beta \pm$ SE for the COPD and asthma group. Regression models were adjusted for age, sex, pack-years, body mass index and type of computed tomography scanner. Pi10: square root of wall area of a 10-mm luminal perimeter. " : additionally adjusted for current smoking status; ": additionally adjusted for clinical centre. 
TABLE 4 Most significant genome-wide association results for the overlap of chronic obstructive pulmonary disease and asthma among non-Hispanic whites

\begin{tabular}{lcccccc} 
Chromosome & SNP & $\begin{array}{c}\text { Gene/nearest } \\
\text { gene }\end{array}$ & $\begin{array}{c}\text { Minor } \\
\text { allele }\end{array}$ & MAF & $\boldsymbol{\beta} \pm$ SE & p-value \\
\hline $\mathbf{8}$ & rs11779254 & CSMD1 & G & 0.42 & $-0.47 \pm 0.10$ & $1.57 \times 10^{-6}$ \\
$\mathbf{1 2}$ & rs59569785 & SOX5 & C & 0.17 & $-0.54 \pm 0.11$ & $1.61 \times 10^{-6}$ \\
$\mathbf{1 2}$ & rs10860172 & RMST & C & 0.33 & $0.50 \pm 0.11$ & $2.55 \times 10^{-6}$ \\
$\mathbf{1 2}$ & rs16926459 & SOX5 & G & 0.84 & $0.52 \pm 0.11$ & $3.39 \times 10^{-6}$ \\
$\mathbf{5}$ & rs72812713 & SEMA6A & T & 0.16 & $-0.52 \pm 0.11$ & $4.48 \times 10^{-6}$ \\
$\mathbf{8}$ & rs6997939 & CSMD1 & C & 0.33 & $-0.44 \pm 0.10$ & $4.53 \times 10^{-6}$ \\
$\mathbf{9}$ & rs4298581 & ZDHHC21 & C & 0.07 & $-0.69 \pm 0.15$ & $5.65 \times 10^{-6}$ \\
$\mathbf{1 2}$ & rs11108963 & RMST & C & 0.31 & $0.51 \pm 0.11$ & $5.66 \times 10^{-6}$ \\
$\mathbf{9}$ & rs10810196 & ZDHHC21 & T & 0.07 & $-0.71 \pm 0.16$ & $5.75 \times 10^{-6}$ \\
$\mathbf{9}$ & rs112457842 & ZDHHC21 & G & 0.07 & $-0.69 \pm 0.15$ & $5.79 \times 10^{-6}$ \\
\hline
\end{tabular}

Genes either contain the variant or are located within $500 \mathrm{~kb}$ of the variant. SNP: single nucleotide polymorphism; MAF: minor allele frequency.

We compared the imaging features between the subjects with COPD and asthma and those with COPD alone, adjusting for age, sex, race, BMI and CT scanner type (table 3). Overlap subjects had less (log) emphysema $(1.44 \pm 1.6 \%$ versus $1.9 \pm 1.4 \%, \mathrm{p}<0.001)$ and more airway disease, measured by wall area of segmental airways $(63.6 \pm 3.3 \mathrm{~mm}$ versus $62.8 \pm 3.0 \mathrm{~mm}, \mathrm{p}<0.001)$ and subsegmental airways $(66.4 \pm 2.7 \mathrm{~mm}$ versus $65.6 \pm 2.3 \mathrm{~mm}, \mathrm{p}=0.001)$, as well as Pi10 $(3.8 \pm 0.2 \mathrm{~mm}$ versus $3.7 \pm 0.1 \mathrm{~mm}$, $\mathrm{p}<0.001)$. There was no significant difference in gas trapping between the two groups.

\section{Genetic analysis}

In a genome-wide analysis of COPD and asthma compared to COPD alone, there were no SNPs that exceeded a genome-wide significance threshold of $5 \times 10^{-8}$ among non-Hispanic whites or AfricanAmerican subjects. Tables 4 and 5 present the most significant variants within each group. The most significant variant among non-Hispanic whites was located within the CSMD1 gene on chromosome 8 $\left(\mathrm{rs} 11779254, \mathrm{p}=1.57 \times 10^{-6}\right)$ (fig. S1). A variant located within an intronic region in the SOX5 gene on chromosome 12 (rs59569785, $\left.\mathrm{p}=1.61 \times 10^{-6}\right)$ was the second most highly ranked SNP (fig. S2).

Among the African-American subjects, there were also several variants that approached genome-wide significance. The most significant SNP ( $\mathrm{rs} 2686829, \mathrm{p}=4.03 \times 10^{-7}$ ) is located in the PKD1L1 gene on chromosome 7. The two top SNPs in the non-Hispanic white population (rs11779254 and rs59569785) that

\begin{tabular}{|c|c|c|c|c|c|c|}
\hline Chromosome & SNP & $\begin{array}{c}\text { Gene/nearest } \\
\text { gene }\end{array}$ & $\begin{array}{l}\text { Minor } \\
\text { allele }\end{array}$ & MAF & OR $(95 \% \mathrm{CI})$ & p-value \\
\hline 7 & rs2686829 & PKD1L1 & $\mathrm{T}$ & 0.14 & $2.41(2.07-2.75)$ & $4.03 \times 10^{-7}$ \\
\hline 13 & rs9577395 & ATP11A & G & 0.08 & $0.35(-0.09-0.78)$ & $1.90 \times 10^{-6}$ \\
\hline 10 & rs3864801 & REEP3 & C & 0.29 & $2.23(1.90-2.56)$ & $1.94 \times 10^{-6}$ \\
\hline 8 & rs12681559 & $N R G 1$ & G & 0.07 & $0.36(-0.06-0.78)$ & $2.25 \times 10^{-6}$ \\
\hline 4 & rs28895885 & $A G A$ & $\mathrm{~T}$ & 0.05 & $0.28(-0.26-0.81)$ & $2.72 \times 10^{-6}$ \\
\hline 10 & rs6479918 & REEP3 & G & 0.32 & $2.06(1.75-2.36)$ & $3.63 \times 10^{-6}$ \\
\hline 1 & rs115905118 & KCNK1 & C & 0.06 & $0.33(-0.14-0.80)$ & $3.79 \times 10^{-6}$ \\
\hline 13 & rs12585036 & ATP11A & $\mathrm{T}$ & 0.09 & $0.40(0.0-0.79)$ & $3.84 \times 10^{-6}$ \\
\hline 4 & rs10213448 & $A G A$ & $T$ & 0.05 & $0.28(-0.25-0.82)$ & $3.90 \times 10^{-6}$ \\
\hline 1 & rs16859141 & KCNK1 & A & 0.06 & $0.33(-0.14-0.80)$ & $4.51 \times 10^{-6}$ \\
\hline
\end{tabular}

Genes either contain the variant or are located within $500 \mathrm{~kb}$ of the variant. SNP: single nucleotide polymorphism; MAF: minor allele frequency. 
TABLE 6 Most significant genome-wide association results for the overlap of chronic obstructive pulmonary disease and asthma in the meta-analysis of non-Hispanic whites and African-Americans

\begin{tabular}{lccccc} 
Chromosome & SNP & $\begin{array}{c}\text { Gene/nearest } \\
\text { gene }\end{array}$ & $\begin{array}{c}\text { Reference } \\
\text { allele }\end{array}$ & OR (95\% Cl) & p-value \\
\hline $\mathbf{1 4}$ & rs6574978 & GPR65 & T & $1.59(0.29-1.76)$ & $1.18 \times 10^{-7}$ \\
$\mathbf{1 4}$ & rs8004567 & GPR65 & A & $0.61(-0.68-0.79)$ & $1.18 \times 10^{-7}$ \\
$\mathbf{1 4}$ & rs1441809 & GPR65 & A & $1.58(0.29-1.76$ & $1.58 \times 10^{-7}$ \\
$\mathbf{1 4}$ & rs3759712 & GALC & C & $0.63(-0.63-0.80)$ & $1.80 \times 10^{-7}$ \\
$\mathbf{1 4}$ & rs6574977 & GPR65 & A & $0.64(-0.62-0.81)$ & $3.16 \times 10^{-7}$ \\
$\mathbf{1 4}$ & rs2165570 & GPR65 & $\mathrm{T}$ & $0.67(-0.56-0.82)$ & $3.95 \times 10^{-7}$ \\
$\mathbf{1 4}$ & rs6574980 & LOC283587 & $\mathrm{T}$ & $1.60(0.29-1.78)$ & $4.48 \times 10^{-7}$ \\
$\mathbf{1 4}$ & $\mathrm{rs} 7157094$ & GPR65 & $\mathrm{T}$ & $0.63(-0.64-0.81)$ & $7.00 \times 10^{-7}$ \\
$\mathbf{8}$ & $\mathrm{rs6471581}$ & CYP11B2 & $\mathrm{T}$ & $0.66(-0.57-0.83)$ & $7.74 \times 10^{-7}$ \\
$\mathbf{8}$ & $\mathrm{rs} 4736359$ & CYP11B2 & $\mathrm{T}$ & $1.51(0.25-1.67)$ & $7.79 \times 10^{-7}$ \\
\hline
\end{tabular}

SNP: single nucleotide polymorphism.

were associated with the overlap status in the non-Hispanic white were not significant in the AfricanAmerican population, although the effect size was in the same direction.

We performed a meta-analysis between the results from the non-Hispanic white and African-American subjects (table 6 and figs S3 and S4). The most significant SNPs were in or near the gene GPR65 on chromosome 14 (fig. 1). These SNPs were located in coding ( $r 6574987, p=1.18 \times 10^{-7}$ ), upstream ( $r$ 8004567, $\mathrm{p}=1.18 \times 10^{-7}$ ) and intronic ( $\left.\mathrm{rs} 14418089, \mathrm{p}=1.58 \times 10^{-7}\right)$ regions, and demonstrated linkage disequilibrium $\left(\mathrm{r}^{2}=0.8-1.0\right)$.

We examined known asthma SNPs for their association with the overlap syndrome (table S5). SNPs in the gene HLA-DQ were nominally significantly associated with COPD and asthma overlap (rs2647012, $\mathrm{p}=0.0056)$ and several additional SNPs were also nominally significant (rs12150298, PERLD1 and rs130065, CCHCR1) $(\mathrm{p}<0.05)$. However, these associations do not meet strict multiple testing correction. We additionally tested known COPD SNPs from the genes HHIP (rs13118928), FAM13a (rs7671167 and rs2869967), CHRNA3/5/IREB2 (rs1051730, rs8034191 and rs2568494), RIN3 (rs754388 and rs17184313) and MMP12 (rs626750 and rs17368814) for their association with the COPD and asthma overlap; none of these SNPs were significantly associated.

\section{Discussion}

In a large study of non-Hispanic white and African-American subjects with COPD, we demonstrate that subjects with both COPD and asthma have more frequent and severe respiratory exacerbations despite
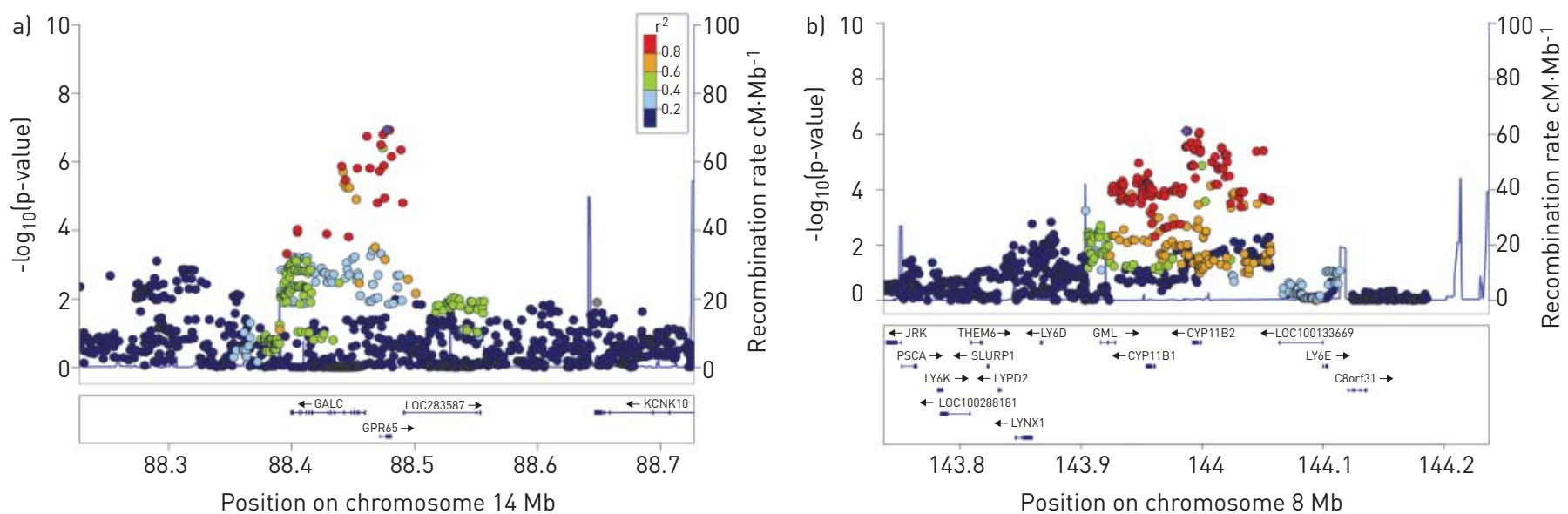

FIGURE 1 Regional association plot from the top meta-analysis results for single nucleotide polymorphisms in or near a) GPR65 (rs6574987) and b) CYP11B2 (rs6471581). cM: centimorgan; Mb: megabases. 
similar lung function and fewer pack-years of smoking exposure compared to subjects with COPD alone. Females and African-Americans were more likely to have both COPD and asthma. We identified imaging differences between these two groups. Subjects with both COPD and asthma demonstrate greater airway wall thickness and less emphysema than subjects with COPD alone. In the genetic analyses, we identified several variants associated with the overlap of COPD and asthma that approach genome-wide significance. The two most significant associations among non-Hispanic white overlap subjects include variants from the CSMD1 gene, which has been associated with emphysema [25], and within the SOX5 gene, which has previously been associated with COPD and may play a role in lung development [26]. In a meta-analysis across the non-Hispanic white and African-American groups, we identified several variants in the GPR65 gene that are associated with the overlap syndrome. To our knowledge, our study is the largest study of smokers with COPD and asthma with such comprehensive imaging and genetic data, and the only study to include genetic information on African-American subjects with COPD and asthma.

In a previous analysis, we investigated the clinical features of this overlap syndrome in the first 2500 subjects enrolled in the COPDGene study, which included 915 subjects with COPD of whom 119 had an additional history of asthma [3]. We demonstrated that subjects with COPD and asthma have more respiratory exacerbations and more severe exacerbations than those with COPD alone, despite similar smoking exposure and lung function. These findings remained significant in our current study, even after removing the initial 915 subjects from analysis (table S1). Our study adds comprehensive chest CT data to investigate differences in airways and parenchyma between these subjects, and allows us to explore shared genetic origins between these two groups [8].

Our current analysis is consistent with prior data suggesting that subjects with both COPD and asthma are at greater risk for respiratory exacerbations. Asthmatic subjects with fixed airway obstruction have demonstrated greater lung function decline and respiratory exacerbations [27]. The presence of more frequent respiratory exacerbations among the overlap group supports the concept that this is a distinct clinical group with greater morbidity [28]. In contrast to our results, a recent study from Spain using the same definitions for COPD/asthma did not demonstrate an increase in exacerbation frequency in the overlap group compared to COPD subjects with either an emphysematous or chronic bronchitic phenotype [29]. However, the sample size was smaller, with fewer females and no African-American subjects.

We demonstrate that both females and African-Americans are more likely to be present in the overlap population. Females and African-Americans have a greater incidence of asthma than non-Hispanic whites and females are often more likely to be diagnosed with asthma than COPD [30]. There is evidence that both females and African-Americans have different COPD susceptibility and clinical presentations than nonHispanic whites, including greater susceptibility to cigarette smoke and worse symptom-related quality of life $[31,32]$. This current study is unique in its ability to characterise features of the overlap syndrome among African-Americans.

Our analysis is one of the first to describe the imaging features of the overlap of COPD and asthma. We demonstrate that subjects with COPD and asthma have less emphysema, but thicker airways than subjects with COPD alone. This data extends on that from prior studies demonstrating distinct pathological and radiographic features among asthmatics with fixed airway disease. Asthmatics who smoke have thicker airway epithelium on biopsy than those who do not, and this thickened epithelium is correlated to respiratory symptoms including increased phlegm production and shortness of breath [33]. When compared to subjects with COPD, asthmatics with fixed airflow obstruction demonstrate less emphysema by CT scan and thicker reticular basement membrane on bronchial biopsy [34]. The radiographic features of thickened airways correlate with histological changes in the small airways [12]. Our findings suggest that the airflow limitation in the overlap subjects may be the result from different pathological changes in the small airways that are different from those among subjects with COPD alone.

We performed a genome-wide association study for genetic variants that could be associated with the COPD/asthma overlap. The "Dutch hypothesis" proposes that COPD and asthma share common genetic risk factors, modified by environmental exposures to produce different clinical phenotypes [8, 35]. The aim of this GWAS was to identify genetic risk factors that are present in the overlap population compared to COPD alone. Although our study is probably underpowered and no markers achieved conventional levels of genome-wide significance $\left(\mathrm{p}<5 \times 10^{-8}\right)$, several of the top SNPs are of interest. Variants located within the CSMD1 gene were among the most significant associations identified in a recent GWAS investigating radiographical features of emphysema [25]. Our group has previously demonstrated several variants downstream from the SOX5 gene were located within a region of genetic linkage and associated with COPD in multiple cohorts. Additionally, the SOX5 knockout mouse has been shown to have abnormal lung development [26]. It is possible that abnormalities in lung development could predispose to fixed airway disease among subjects with asthma, suggesting a common genetic origin of asthma and COPD. Among the 
African-American subjects, we did not identify any significant associations with the overlap of COPD and asthma, probably related to the smaller sample size of this population.

In a meta-analysis of non-Hispanic whites and African-Americans, we identified several coding and noncoding SNPs in the GPR65 gene that were associated with the overlap syndrome. The SNP with the lowest p-value approached genome-wide significance. The protein product of GPR65 is a member of the G2A G protein-coupled receptor family and plays an important role in eosinophil activation during asthma and extracellular inhibition of proinflammatory cytokines [36]. This protein is expressed in eosinophils and plays a role in acid-mediated eosinophil viability. Gpr65 knock-out mice have demonstrated attenuated airway eosinophilia in murine asthma models [37]. Future studies with larger samples of genotyped subjects with COPD and asthma may reveal a significant role for this gene in COPD and asthma overlap.

Our study has several limitations. The COPDGene Study enrolled adult smokers with and without COPD, and is one of the few large COPD studies that did not exclude subjects with asthma. We based our analysis of asthma on self-reported history of asthma, which could be subject to recall bias or physician misdiagnosis. We used an age limit of 40 years for diagnosis in an attempt to better capture early-onset asthma. Using this definition, we were able to identify a distinct population that demonstrated both clinical and imaging features that are different to those of subjects with COPD alone. In addition, these subjects have more hay fever and are more likely to have a parental history of asthma, lending validity to the distinct nature of this group. Of note, there was no significant difference in the number of subjects exhibiting a response to bronchodilator between the two groups, highlighting the limited ability of this trait to identify asthma in COPD. This is a cross-sectional analysis and, therefore, we are limited in our ability to examine the long-term outcomes. However, as a surrogate, the higher BODE score suggests that the COPD and asthma overlap subjects may have greater risk of mortality. Future longitudinal studies in this population may better demonstrate differential clinical outcomes between these populations.

The GWAS found interesting associations with biologically plausible genes, but did not identify any genome-wide significant results. This is probably a result of our relatively small sample size compared to GWAS of other chronic diseases. The genetic architecture of the overlap group is probably similar to other complex traits, with multiple markers of small effect size being involved. Unfortunately, as COPDGene is unique in its inclusion of a large proportion of COPD subjects with a history of asthma, there is not an adequately sized cohort to enable us to replicate our genetic findings.

Our study has potential clinical implications. Although the subjects in our study were primarily identified on the basis of their spirometry, spirometry is less common in outpatient clinics and is often underutilised. Our study identified a group of subjects with asthma and fixed airway obstruction. It is important for physicians to consider the presence of fixed airway disease, especially among asthmatic patients who smoke. Additionally, the presence of distinct radiographic features in this group, with features that are more consistent with asthma, suggests that treatment plans for these patients incorporating aspects of asthma management such as earlier use of inhaled corticosteroids could potentially be beneficial. Finally, we identified that more females and African-Americans have COPD and asthma overlap. Clinicians should be alert to the possible coexistence of both disorders in all patients, but especially these populations.

In summary, we defined a subgroup of subjects who have COPD and asthma. These subjects demonstrate distinct clinical features compared to those with COPD alone. Subjects with COPD and asthma are an important clinical population that may have distinct genetic risk factors. Longitudinal follow-up in COPDGene will characterise disease progression. Future therapeutic studies are needed to identify optimal treatment approaches for patients with concurrent asthma and COPD.

\section{Acknowledgements}

We would like to acknowledge and thank the COPDGene Core Teams listed below.

Administrative Core (Boston, MA, USA and Denver, CO, USA): J. Crapo (Principal Investigator), E. Silverman (Principal Investigator), B. Make, E. Regan, R. Lantz, L. Stepp and S. Melanson. Genetic Analysis Core (Boston, MA, USA, Balitmore, MA, USA and Denver, CO, USA): T. Beaty, B. Klanderman, N. Laird, C. Lange, M. Cho, S. Santorico, J. Hokanson, D. DeMeo, N. Hansel, C. Hersh, P. Castaldi, M-L. McDonald, J. Zhou, M. Mattheissen, E. Wan, M. Hardin, J. Hetmanski, M. Parker and T. Murray. Imaging Core (Boston, MA, USA and Denver, CO, USA): D. Lynch, J. Schroeder, J. Newell Jr, J. Reilly, H. Coxson, P. Judy, E. Hoffman, G. Washko, R. San Jose Estepar, J. Ross, M. Al Qaisi, J. Zach, A. Kluiber, J. Sieren, T. Mann, D. Richert, A. McKenzie, J. Akhavan and D. Stinson. PFT QA Core, LDS Hospital, Salt Lake City, UT, USA: R. Jensen. Biological Repository, Johns Hopkins University, Baltimore, MD, USA: H. Farzadegan, S. Meyerer, S. Chandan and S. Bragan. Data Coordinating Center and Biostatistics, National Jewish Health, Denver, CO, USA: D. Everett, A. Williams, C. Wilson, A. Forssen, A. Powell and J. Piccoli. Epidemiology Core, University of Colorado School of Public Health, Denver, CO, USA: J. Hokanson, M. Sontag, J. Black-Shinn, G. Kinney and S. Lutz.

We also wish to acknowledge the COPDGene Investigators from the following participating clinical centres. VA Ann Arbor Healthcare System, Ann Arbor, MI, USA: J. Curtis and E. Kazerooni. Baylor College of Medicine, Houston, TX, 
USA: N. Hanania, P. Alapat, V. Bandi, K. Guntupalli, E. Guy, A. Mallampalli, C. Trinh, M. Atik, H. Al-Azzawi, M. Willis, S. Pinero, L. Fahr, A. Nachiappan, C. Bray, L. Alexander Frigini, C. Farinas, D. Katz, J. Freytes and A.M. Marciel. Brigham and Women's Hospital, Boston, MA, USA: D. DeMeo, C. Hersh, G. Washko, F. Jacobson, H. Hatabu, P. Clarke, R. Gill, A. Hunsaker, B. Trotman-Dickenson and R. Madan. Columbia University, New York, NY, USA: R.G. Barr, B. Thomashow, J. Austin and B. D'Souza. Duke University Medical Center, Durham, NC, USA: N. MacIntyre Jr, L. Washington and H.P. McAdams. Fallon Clinic, Worcester, MA, USA: R. Rosiello, T. Bresnahan, J. Bradley, S. Kuong, S. Meller and S. Roland. Health Partners Research Foundation, Minneapolis, MN, USA: C. McEvoy and J. Tashjian. Johns Hopkins University, Baltimore, MD, USA: R. Wise, N. Hansel, R. Brown, G. Diette and K. Horton. Los Angeles Biomedical Research Institute at Harbor UCLA Medical Center, Los Angeles, CA, USA: R. Casaburi, J. Porszasz, H. Fischer, M. Budoff and M. Rambod. Michael E. DeBakey Veterans Affairs Medical Center, Houston, TX, USA: A. Sharafkhaneh, C. Trinh, H. Kamal, R. Darvishi, M. Willis, S. Pinero, L. Fahr, A. Nachiappan, C. Bray, L.A. Frigini, C. Farinas, D. Katz, J. Freytes and A.M. Marciel. Minneapolis VA Health Care System, Minneapolis, MN, USA: D. Niewoehner, Q. Anderson, K. Rice and A. Caine. Morehouse School of Medicine, Atlanta, GA, USA: M. Foreman, G. Westney and E. Berkowitz. National Jewish Health, Denver, CO, USA: R. Bowler, D. Lynch, J. Schroeder, V. Hale, J. Armstrong II, D. Dyer, J. Chung and C. Cox. Temple University, Philadelphia, PA, USA: G. Criner, V. Kim, N. Marchetti, A. Satti, A.J. Mamary, R. Steiner, C. Dass and L. Cone. University of Alabama, Birmingham, AL, USA: W. Bailey, M. Dransfield, M. Wells, S. Bhatt, H. Nath and S. Singh. University of California San Diego, La Jolla, CA, USA: J. Ramsdell and P. Friedman. University of Iowa, Iowa City, IA, USA: A. Cornellas, J. Newell Jr and E.J.R. van Beek. University of Michigan, Ann Arbor, MI, USA: F. Martinez, M. Han and E. Kazerooni. University of Minnesota, Minneapolis, MN, USA: C. Wendt and T. Allen. University of Pittsburgh, Pittsburgh, PA, USA: F. Sciurba, J. Weissfeld, C. Fuhrman, J. Bon and D. Hooper. University of Texas Health Science Center at San Antonio, San Antonio, TX, USA: A. Anzueto, S. Adams, C. Orozco, M. Ruiz, A. Mumbower, A. Kruger, C. Restrepo and M. Lane.

\section{References}

1 Soriano JB, Davis KJ, Coleman B, et al. The proportional Venn diagram of obstructive lung disease: two approximations from the United States and the United Kingdom. Chest 2003; 124: 474-481.

2 de Marco R, Pesce G, Marcon A, et al. The coexistence of asthma and chronic obstructive pulmonary disease (COPD): prevalence and risk factors in young, middle-aged and elderly people from the general population. PLoS One 2013; 8: e62985.

3 Hardin M, Silverman EK, Barr RG, et al. The clinical features of the overlap between COPD and asthma. Respir Res 2011; 12: 127.

4 Miravitlles M, Soler-Cataluna JJ, Calle M, et al. A new approach to grading and treating COPD based on clinical phenotypes: summary of the Spanish COPD guidelines (GesEPOC). Prim Care Respir J 2013; 22: 117-121.

5 O’Donnell DE, Aaron S, Bourbeau J, et al. Canadian Thoracic Society recommendations for management of chronic obstructive pulmonary disease - 2007 update. Can Respir J 2007; 14: Suppl. B, 5B-32B.

6 Nagai A. [Guidelines for the diagnosis and management of chronic obstructive pulmonary disease: 3rd edition]. Nihon Rinsho 2011; 69: 1729-1734.

7 Meyers DA, Larj MJ, Lange L. Genetics of asthma and COPD. Similar results for different phenotypes. Chest 2004; 126: Suppl. 2, 105S-110S.

8 Postma DS, Kerkhof M, Boezen HM, et al. Asthma and chronic obstructive pulmonary disease: common genes, common environments? Am J Respir Crit Care Med 2011; 183: 1588-1594.

9 Regan EA, Hokanson JE, Murphy JR, et al. Genetic epidemiology of COPD (COPDGene) study design. COPD 2010; 7: 32-43.

10 Miller MR, Hankinson J, Brusasco V, et al. Standardisation of spirometry. Eur Respir J 2005; 26: 319-338.

11 Fedorov A, Beichel R, Kalpathy-Cramer J, et al. 3D slicer as an image computing platform for the quantitative imaging network. Magn Reson Imaging 2012; 30: 1323-1341.

12 Nakano Y, Wong JC, de Jong PA, et al. The prediction of small airway dimensions using computed tomography. Am J Respir Crit Care Med 2005; 171: 142-146.

13 Kim YI, Schroeder J, Lynch D, et al. Gender differences of airway dimensions in anatomically matched sites on CT in smokers. COPD 2011; 8: 285-292.

14 Busacker A, Newell JD Jr, Keefe T, et al. A multivariate analysis of risk factors for the air-trapping asthmatic phenotype as measured by quantitative CT analysis. Chest 2009; 135: 48-56.

15 Zhou X, Baron RM, Hardin M, et al. Identification of a chronic obstructive pulmonary disease genetic determinant that regulates HHIP. Hum Mol Genet 2012; 21: 1325-1335.

16 Howie B, Fuchsberger C, Stephens M, et al. Fast and accurate genotype imputation in genome-wide association studies through pre-phasing. Nat Genet 2012; 44: 955-959.

17 Li Y, Willer CJ, Ding J, et al. MaCH: using sequence and genotype data to estimate haplotypes and unobserved genotypes. Genet Epidemiol 2010; 34: 816-834.

18 Price AL, Patterson NJ, Plenge RM, et al. Principal components analysis corrects for stratification in genome-wide association studies. Nat Genet 2006; 38: 904-909.

19 Purcell S, Neale B, Todd-Brown K, et al. PLINK: a tool set for whole-genome association and population-based linkage analyses. Am J Hum Genet 2007; 81: 559-575.

20 Willer CJ, Li Y, Abecasis GR. METAL: fast and efficient meta-analysis of genomewide association scans. Bioinformatics 2010; 26: 2190-2191.

21 Johnson AD, Handsaker RE, Pulit SL, et al. SNAP: a web-based tool for identification and annotation of proxy SNPs using HapMap. Bioinformatics 2008; 24: 2938-2939.

22 Moffatt MF, Gut IG, Demenais F, et al. A large-scale, consortium-based genomewide association study of asthma. N Engl J Med 2010; 363: 1211-1221.

23 Torgerson DG, Ampleford EJ, Chiu GY, et al. Meta-analysis of genome-wide association studies of asthma in ethnically diverse North American populations. Nat Genet 2011; 43: 887-892.

24 Foreman MG, Campos M, Celedon JC. Genes and chronic obstructive pulmonary disease. Med Clin North Am 2012; 96: 699-711. 

emphysema. Am J Respir Crit Care Med 2011; 183: 43-49. Hersh CP, Silverman EK, Gascon J, et al. SOX5 is a candidate gene for chronic obstructive pulmonary disease susceptibility and is necessary for lung development. Am J Respir Crit Care Med 2011; 183: 1482-1489.

27 Contoli M, Baraldo S, Marku B, et al. Fixed airflow obstruction due to asthma or chronic obstructive pulmonary disease: 5-year follow-up. J Allergy Clin Immunol 2010; 125: 830-837.

28 Hurst JR, Vestbo J, Anzueto A, et al. Susceptibility to exacerbation in chronic obstructive pulmonary disease. N Engl J Med 2010; 363: 1128-1138.

29 Izquierdo-Alonso JL, Rodriguez-Gonzalezmoro JM, de Lucas-Ramos P, et al. Prevalence and characteristics of three clinical phenotypes of chronic obstructive pulmonary disease (COPD). Respir Med 2013; 107: 724-731.

30 Chapman KR, Tashkin DP, Pye DJ. Gender bias in the diagnosis of COPD. Chest 2001; 119: $1691-1695$.

31 Dransfield MT, Davis JJ, Gerald LB, et al. Racial and gender differences in susceptibility to tobacco smoke among patients with chronic obstructive pulmonary disease. Respir Med 2006; 100: 1110-1116.

32 Chatila WM, Hoffman EA, Gaughan J, et al. Advanced emphysema in African-American and white patients: do differences exist? Chest 2006; 130: 108-118.

33 Broekema M, ten Hacken NH, Volbeda F, et al. Airway epithelial changes in smokers but not in ex-smokers with asthma. Am J Respir Crit Care Med 2009; 180: 1170-1178.

34 Fabbri LM, Romagnoli M, Corbetta L, et al. Differences in airway inflammation in patients with fixed airflow obstruction due to asthma or chronic obstructive pulmonary disease. Am J Respir Crit Care Med 2003; 167: 418-424.

35 Postma DS, Boezen HM. Rationale for the Dutch hypothesis. Allergy and airway hyperresponsiveness as genetic factors and their interaction with environment in the development of asthma and COPD. Chest 2004; 126: Suppl. 2, 96S-104S.

36 Mogi C, Tobo M, Tomura H, et al. Involvement of proton-sensing TDAG8 in extracellular acidification-induced inhibition of proinflammatory cytokine production in peritoneal macrophages. J Immunol 2009; 182: 3243-3251.

37 Kottyan LC, Collier AR, Cao KH, et al. Eosinophil viability is increased by acidic pH in a cAMP- and GPR65dependent manner. Blood 2009; 114: 2774-2782. 